\title{
MEASURING REAL-FINANCIAL CONNECTEDNESS IN THE U.S. ECONOMY
}

\author{
Erhan Uluceviz \\ Kamil Y1lmaz
}

Working Paper 1812

September 2018

This Working Paper is issued under the supervision of the ERF Directorate. Any opinions expressed here are those of the author(s) and not those of the Koç University-TÜSİAD Economic Research Forum. It is circulated for discussion and comment purposes and has not been subject to review by referees.

KOÇ UNIVERSITY-TÜSİAD ECONOMIC RESEARCH FORUM

Rumelifeneri Yolu 34450 Sariyer/Istanbul 


\title{
Measuring Real-Financial Connectedness in the U.S. Economy*
}

\author{
Erhan Uluceviz ${ }^{\dagger}$ \\ Gebze Technical University
}

Kamil Yilmaz

Koç University

August 25, 2018

\begin{abstract}
We analyze connectedness between the real and financial sectors of the U.S. economy. Using the weekly ADS index of the Philadelphia FED (the widely used business conditions indicator) to represent the real side, we find that during times of financial distress and/or business cycle turning points the direction of connectedness runs from the real sector to financial markets. The ADS index is derived from a model containing a measure of term structure along with real variables, therefore, it might not be the best representative of the real activity to be used in the connectedness analysis. As an alternative, we derive a real activity index (RAI) from a dynamic factor model of the real sector variables only. The behavior of RAI over time is quite similar to that of the ADS index. When we include RAI to represent the real side of the economy in the connectedness analysis, the direction of net connectedness reverses: financial markets generate positive net connectedness to the real side of the economy.
\end{abstract}

Keywords: Macro-financial linkages, Connectedness, ADS index, Dynamic factor model, Volatility, Vector autoregression, Variance decomposition.

JEL codes: C38, E44, E37, G10.

*We thank the Scientific and Technological Research Council of Turkey (TUBITAK) for financial support through Grant No. 114K954. The usual disclaimer applies.

$\dagger$ e-mail: erhan@uluceviz.com

$\ddagger$ e-mail: kyilmaz@ku.edu.tr (corresponding author) 


\section{Introduction}

In the current global economic context, the issue of real-financial connectedness appears to be of central importance, determining how financial shocks affect the real side of the economy and how shocks to real (macroeconomic) fundamentals may in turn influence financial markets. A better understanding of real-financial connectedness may therefore provide valuable insights in designing policies to prevent financial crises and avoid the economic, political and social consequences of these crises. Despite the obvious importance of the issue from both an academic and policy perspective; however, the links between the real and the financial sides of the economy have remained largely unstudied in the literature. The current study adds to the small literature on this topic with a combination of methodological and empirical contributions.

This paper builds on Diebold-Yilmaz Connectedness Index (DYCI) methodology to investigate the joint structure of connectedness between the real and the financial sides of the U.S. economy. The DYCI methodology is a flexible, and a straightforward-toimplement approach to study interconnectedness between many variables of interest. It has been used extensively to study the structure of financial and macroeconomic connectedness separately. However, applying the DYCI approach to study connectedness across the real and the financial sides of the economy raises additional issues. Most notably, real/macroeconomic data are typically available at much lower frequencies than financial data, but the existing DYCI methodology is only applicable for data observed at a common sampling frequency. Macroeconomic data are usually released in weekly, monthly, quarterly, semi-annual and annual frequencies, whereas financial data could be obtained for every minute, second or even transaction. DYCI analysis is not feasible without circumventing the frequency mismatch in the data.

In this paper, we circumvent the frequency mismatch problem by employing a business conditions index, which is produced by distilling the raw data that are in different frequencies into a common frequency. ${ }^{1}$ To be more specific, we first use the readily available ADS index (see Aruoba et al., 2009) which is generated from daily financial and weekly, monthly and quarterly macroeconomic variables for the U.S. to track business conditions

\footnotetext{
${ }^{1}$ Cotter et al. (2017) applied the DYCI methodology in a mixed-frequency modelling environment to analyze the connectedness of weekly financial variables with monthly real/macroeconomic variables.
} 
on a daily basis. We conduct a weekly analysis, estimate total and directional dynamic connectedness measures and find that ADS acts as a net connectedness source towards financial markets during some critical episodes of the U.S. economy. However, simultaneous use of financial and macroeconomic variables in the estimation of the ADS index prevents it to be a sole performance measure of the real side of the economy. Rather, it is a general measure tracking the performance of the economy as a whole.

To avoid possible complications stemming from the use of a financial variable in the construction of the ADS index, we propose a dynamic factor model based business conditions index à la Aruoba et al. (2009), which tracks real macroeconomic activity with weekly sampling frequency. We employ a weekly dynamic factor model, which is an extension of the monthly model as detailed in Banbura et al. (2011), Banbura and Modugno (2014) and recently used by Scotti (2016). We estimate a weekly real activity index (RAI) from the same set of quarterly, monthly and weekly macroeconomic variables as used in the ADS index and extract the business conditions of the real side of the economy. When compared; RAI closely tracks the ADS index and captures all major economic incidents over time. Once the ADS index is replaced with RAI, we observe the reversal of the roles of the real and financial sides in the connectedness analysis. U.S. financial markets become net generators of connectedness towards the real side. Therefore, financial data in the construction of the ADS index seems to be responsible for this reversal. Strict isolation of the real and the financial sides unveils the dynamics of the connectedness between the real and the financial sides of the U.S. economy.

To represent financial side of the U.S. economy, we calculate weekly Garman-Klass type range-based return volatilities for the stock, government bond (10-year) and the foreign currency markets. To the best of our knowledge, we are the first to extend this type of monthly dynamic factor model to a weekly one and study the interaction of the real and the financial sides of the U.S. economy.

The paper continues with a brief review of the literature on how the real and financial sides of the economy interact in Section 2. Section 3 provides an overview of the DYCI framework used to understand how shocks spread across a system of variables. Section 4 provides empirical results for the real-financial connectedness using the ADS index as the representative of the real side of the U.S. Economy. Section 5 details the construction of 
a weekly real activity index from a dynamic factor model which extracts the real activity indicator, i.e. RAI, we use to represent the real side of the U.S. economy. Section 6 compares the ADS index and RAI as indicators of the U.S. economic activity. Section 7 presents empirical real-financial connectedness results when RAI is used instead of the ADS index to capture the developments in the real side of the U.S. economy. Section 8 compares the connectedness results carried out with the ADS index (Section 4) and RAI (Section 7), respectively. Section 9 concludes the paper.

\section{Literature Review}

In recent decades, markets on the real and financial sides of the economy have become increasingly interdependent, both within and across national borders. This increase in connectedness allows idiosyncratic economic or financial shocks in particular markets to be amplified and transmitted easier to other markets, thus exposing nations to increasing socioeconomic risk on a global scale. The danger this presents was clearly demonstrated by the recent 2008-2009 financial and economic crisis, which has lead to a dramatic increase in research activity on market connectedness and systemic risk.

Most notably, a large literature has been created on the subject of modelling and measuring connectedness or systemic stress within the financial side of the economy. Recent work on this subject can be divided roughly into two groups, with the first consisting of approaches aiming to model and explain interconnectedness between financial institutions using structural models. Many recent examples employ network models, which provide a natural way to model a group of individual financial institutions and the bilateral assets or liabilities between them. Recent examples of such network-based methods include Gai and Kapadia (2010), Gai et al. (2011), Markose et al. (2012), Acemoglu et al. (2015), and Anand et al. (2013). The second branch of the literature derives statistical measures for financial connectedness and systemic risk that are typically non-structural in nature. Notable examples include the CoVaR approach of Adrian and Brunnermeier (2011), the volatility spillover framework of Diebold and Yilmaz (2009, 2012, 2014), the systemic expected shortfall measure of Acharya et al. (2010), the dynamic equicorrelation model of Engle and Kelly (2012), and the measures proposed by Billio et al. (2012). 
This recent research on financial connectedness has provided many new and important insights into connectedness and systemic risk in financial markets and dramatically improved our understanding of this important topic. The theoretical and empirical results of these studies also have direct policy implications for monetary authorities, financial regulators, national governments and international organisations such as the IMF. In particular, structural models can potentially help regulators to understand whether certain financial network structures are more vulnerable to systemic events (see, for example Acemoglu et al., 2015), or to identify the factors under regulatory control that affect the predicted losses caused by crisis events (see, for example Gai et al., 2011). The non-structural measures of connectedness can be used to identify systemically important financial institutions and the key channels via which shocks are transmitted through the financial system. Based on this information, regulatory policy can be adjusted to minimise the vulnerability of the financial system to adverse shocks.

Outside of this work on financial market connectedness, a small number of studies have also examined connectedness within the real side of the economy, such as Acemoglu et al. (2012) and Diebold and Yilmaz (2015). However, discussions of systemic risk frequently emphasize the potential for systemic stress in financial markets to adversely affect the real side of the economy and the possibility that macroeconomic shocks may in turn trigger periods of systemic stress in financial markets (see, for example Borio and Drehmann, 2011; Brunnermeier et al., 2011, Blancher et al., 2013). Given the obvious importance of this issue, it is surprising that the links between the real and financial sides of the economy have received little attention and remain largely unstudied. A small number of notable exceptions that do study connectedness on both the financial and real sides of the economy in some form include He and Krishnamurthy (2012), Anand et al. (2013), Dungey et al. (2013), Barattieri et al. (2013) and Cotter et al. (2017). Whilst making contributions to the subject, none of the above cited studies, perhaps with the exception of Cotter et al. (2017), have modeled these macro-financial relationships in a complete or entirely satisfactory manner, leaving room for much work to be done. Applying DYCI methodology in a mixed-frequency environment, Cotter et al. (2017) show that financial markets are typically net transmitters of shocks to the real side of the economy, particularly during turbulent market conditions. Our work is a modest attempt 
to study the issue from a slightly different angle than Cotter et al. (2017).

On the other hand, treating real activity of the economy as a single series has been a central theme to many approaches to business cycle analysis starting from Burns and Mitchell (1946). There is an important body of literature which extracts latent business conditions using a dynamic factor model (see, for example Stock and Watson, 1989, 1991; Mariano and Murasawa, 2003; Aruoba et al., 2009). Extracted latent factor is usually closely followed by central banks for nowcasting GDP or tracking the health of the economy, (see, for example Banbura et al., 2011; Aruoba et al., 2009). Limits on the use of the extracted latent factor as a real activity index extends the aforementioned applications, see e.g. Scotti (2016) who generates surprise index to "measure agents' optimism or pessimism about the economy" through the use of real activity index series.

\section{DYCI Methodology}

This section provides a summary of the connectedness index methodology, which was developed in a series of papers, see Diebold and Yilmaz (2009, 2012, 2014). Diebold and Yilmaz (2014) showed that the connectedness framework was closely linked with the modern network theory. They showed that the total connectedness measure corresponds to the mean degree of a weighted, directed network where variables represent the nodes of the network.

The connectedness index is built upon the variance decomposition matrix associated with an $N$-variable vector autoregression. The total connectedness index is the ratio of the sum of off-diagonal elements of the forecast error variance-covariance matrix to the sum of all elements of the same matrix.

Consider a covariance stationary $N$-variable $\operatorname{VAR}(p), x_{t}=\sum_{i=1}^{p} \Phi_{i} x_{t-i}+\varepsilon_{t}$, where $\varepsilon_{t} \sim(0, \Sigma)$. The moving average (MA) representation is $x_{t}=\sum_{i=0}^{\infty} A_{i} \varepsilon_{t-i}$, where the $N \mathrm{x} N$, coefficient matrices $A_{i}$ obey the recursion $A_{i}=\Phi_{1} A_{i-1}+\Phi_{2} A_{i-2}+\ldots+\Phi_{p} A_{i-p}$, with $A_{0}$ an $N \mathrm{x} N$ identity matrix and $A_{i}=0$ for $i<0$. The moving average coefficients (or transformations such as impulse response functions or variance decompositions) are the key to understanding the dynamics. We rely on variance decompositions, which allows us to split the forecast error variances of each variable into parts attributable to 
the various system shocks. Variance decompositions also allow one to assess the fraction of the $H$-step-ahead error variance in forecasting $x_{i}$ that is due to shocks to $x_{j}, \forall i \neq j$, for each $i$.

A study of connectedness is not complete without an analysis of directional connectedness across the nodes. Calculation of variance decompositions requires orthogonal innovations, whereas the VAR innovations are generally correlated. Identification schemes such as that based on Cholesky factorization achieve orthogonality, but the variance decompositions then depend on the ordering of the variables. As a result, it is not possible to use the variance decompositions from the Cholesky factor orthogonalization to study the direction of connectedness. With this understanding, Diebold and Yilmaz (2012) propose to circumvent this problem by exploiting the generalized VAR framework of Koop et al. (1996) and Pesaran and Shin (1998), which produces variance decompositions invariant to ordering. Instead of attempting to orthogonalize shocks, the generalized approach allows correlated shocks but accounts for them appropriately using the historically observed distribution of the errors. As the shocks to each variable are not orthogonalized, the sum of contributions to the variance of forecast error (that is, the row sum of the elements of the variance decomposition table) is not necessarily equal to one.

Using the VAR framework introduced above, we define own variance shares to be the fractions of the $H$-step-ahead error variances in forecasting $x_{i}$ due to shocks to $x_{i}$, for $i=1,2, . ., N$, and cross variance shares, or connectedness, to be the fractions of the $H$-step-ahead error variances in forecasting $x_{i}$ due to shocks to $x_{j}$, for $i, j=1,2, . ., N$, such that $i \neq j$.

The generalized impulse response and variance decomposition analyses also rely on the MA representation of the $N$-variable $\operatorname{VAR}(p)$ equation above. Pesaran and Shin (1998) show that when the error term $\varepsilon_{t}$ has a multivariate normal distribution, the $h$ step generalized impulse response function scaled by the variance of the variable is given by:

$$
\gamma_{j}^{g}(h)=\frac{1}{\sqrt{\sigma_{j j}}} A_{h} \Sigma \boldsymbol{e}_{j}, \quad h=0,1,2, \ldots
$$

where $\Sigma$ is the variance matrix for the error vector $\varepsilon, \sigma_{j j}$ is the standard deviation of the 
error term for the $j^{\text {th }}$ equation and $\boldsymbol{e}_{i}$ is the selection vector with one as the $i^{\text {th }}$ element and zeros otherwise. Node $j$ 's contribution to node $i$ 's $H$-step-ahead generalized forecast error variance, $\theta_{i j}^{g}(H)$, for $H=1,2, \ldots$, is defined as:

$$
\theta_{i j}^{g}(H)=\frac{\sigma_{j j}^{-1} \sum_{h=0}^{H-1}\left(e_{i}^{\prime} A_{h} \Sigma e_{j}\right)^{2}}{\sum_{h=0}^{H-1}\left(e_{i}^{\prime} A_{h} \Sigma A_{h}^{\prime} e_{i}\right)}
$$

As explained above, the sum of the elements of each row of the variance decomposition table is not necessarily equal to one, i.e. $\sum_{j=1}^{N} \theta_{i j}^{g}(H) \neq 1$. In order to use the information available in the variance decomposition matrix to calculate the connectedness index, Diebold and Yilmaz (2012) normalize each entry of the variance decomposition matrix, equation 2 , by the row sum $\mathrm{as}^{2}$ :

$$
C_{i \leftarrow j}^{H}=\frac{\theta_{i j}^{g}(H)}{\sum_{j=1}^{N} \theta_{i j}^{g}(H)}
$$

Now, by construction $\sum_{j=1}^{N} C_{i \leftarrow j}^{H}=1$ and $\sum_{i, j=1}^{N} C_{i \leftarrow j}^{H}=N$. Using the normalized entries of the generalized variance decomposition matrix, equation 3, Diebold and Yilmaz (2012) construct the total connectedness index as:

$$
C^{H}=\frac{\sum_{\substack{i, j=1 \\ i \neq j}}^{N} C_{i \leftarrow j}^{H}}{\sum_{i, j=1}^{N} C_{i \leftarrow j}^{H}}=\frac{\sum_{\substack{i, j=1 \\ i \neq j}}^{N} C_{i \leftarrow j}^{H}}{N}
$$

Next considering directional connectedness, Diebold and Yilmaz (2012) define gross directional connectedness received by node $i$ from all other nodes $j$ as:

$$
C_{i \leftarrow \bullet}^{H}=\frac{\sum_{\substack{j=1 \\ j \neq i}}^{N} C_{i \leftarrow j}^{H}}{\sum_{i, j=1}^{N} C_{i \leftarrow j}^{H}} \times 100=\frac{\sum_{\substack{j=1 \\ j \neq i}}^{N} C_{i \leftarrow j}^{H}}{N} \times 100
$$

In a similar fashion, directional volatility connectedness transmitted from node $i$ to all other nodes $j$ is measured as:

$$
C_{\bullet \leftarrow i}^{H}=\frac{\sum_{\substack{j=1 \\ j \neq i}}^{N} C_{j \leftarrow i}^{H}}{\sum_{i, j=1}^{N} C_{j \leftarrow i}^{H}} \times 100=\frac{\sum_{\substack{j=1 \\ j \neq i}}^{N} C_{j \leftarrow i}^{H}}{N} \times 100
$$

\footnotetext{
${ }^{2}$ Alternatively, one can normalize the elements of the variance decomposition matrix with the column sum of these elements and compare the resulting total connectedness index with the one obtained from the normalization with the row sum.
} 
One can think of the set of directional connectedness as providing a decomposition of total connectedness into those transmitted by each node in the sample. Obviously, once the shocks transmitted and received by node $i$ are calculated, the difference between the two will result in a measure of the net directional connectedness transmitted from node $i$ to all other nodes as:

$$
C_{i}^{H}=C_{\bullet \leftarrow i}^{H}-C_{i \leftarrow \bullet}^{H}
$$

The net directional connectedness index, equation 7, provides information about how much volatility each node contributes in net terms to other nodes, respectively.

\section{Measuring Real-Financial Connectedness with ADS}

In this section, we investigate connectedness between the real and financial sectors of the U.S. economy. We use the Aruoba-Diebold-Scotti (ADS) index to represent the real activity in the U.S. economy. ADS index is a business conditions indicator developed by Aruoba et al. (2009). It is designed to track economic activity at daily frequency. It is published regularly by Philadelphia FED and is available since March 1, $1960 .{ }^{3}$

We use weekly return volatilities for S\&P 500 stock market index (SPX), 10-year government bonds (US10YT) and U.S. dollar index (DXY) to represent the financial side of the U.S. economy. Volatilities are computed as in Diebold and Yilmaz (2009), i.e., through a Garman-Klass type range-based method.

Empirical implementation of the DYCI framework is based on the estimation of a VAR(3) model, over 4-year (208-week) rolling sample windows for the period from January 1980 to June 2016. We choose a 52-week forecast horizon. For some sample windows, the estimated VAR model can be nonstationary. When that is the case, connectedness index becomes unreliable. For that reason, Figure 1 presents only the total connectedness results when the estimated VAR model is stationary. Throughout our presentation, shaded regions in the plots represent U.S. recession dates.

From the 1980s until the global financial crisis of 2008-09, the real-financial connect-

\footnotetext{
${ }^{3}$ For further details of the ADS index, see https://www.philadelphiafed.org/research-and-data/realtime-center/business-conditions-index, retrieved 2018-01-28.
} 


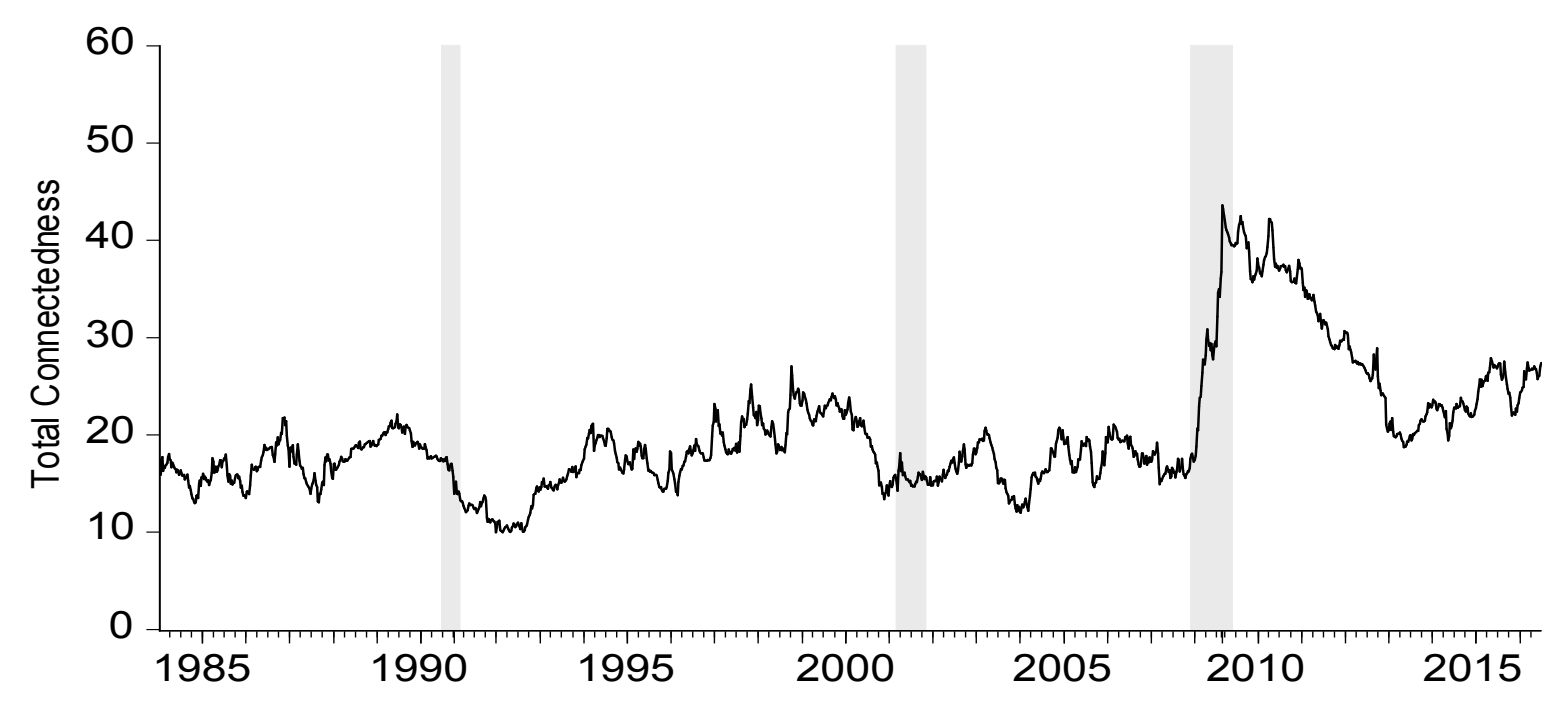

Figure 1: Total Connectedness Index using ADS

edness (as measured with the ADS index) fluctuates within the 10-25\% band. The total connectedness index shows slight (less than 10\%) jumps following the stock market crash of October 19, 1987 (so-called Black Monday), East Asian financial crisis of 1997 and the collapse of LTCM hedge fund in September 1998 following the Russian financial crisis. The index also shows a gradual move from around 10\% in 1992 to around $20 \%$ in 1994. As far as we know, there was no major development in the U.S. real and financial sides U.S. economy during this period. The only possibility for this period could be the positive reaction of the financial markets to the expected North American Free Trade agreement. The only significant development in international markets during this period was the Exchange Rate Mechanism (ERM) crisis in Europe that took place in September 1992 which had repurcussions until August 1993.

With the 2008-09 global financial crisis, total connectedness between the real and financial sides of the U.S. economy reaches its maximum level over our analysis period (see Figure 1). Due to the ensuing European sovereign debt crisis of 2010-2012, the index stayed at a high plateau for a while. Following this unprecedented period of turmoil in international financial markets, total connectedness index has stabilized and stayed within the $20-30 \%$ band. We also observe slight upward moves in 2013-2015 period 
reflecting the effects of Taper Tantrum ${ }^{4}$ (June 2013), bond market flash crash (October 2014) and increased worries about the Chinese financial markets (summer 2015).

The DYCI framework allows us to further investigate the sources of shocks that lead to increases in connectedness across the real and financial sectors. In Figure 2, we plot the "to others", "from others" and "net" directional connectedness measures for each node in the network. We observe from the last row of Figure 2 that the real side of the U.S. economy acts as a source net of connectedness except for certain sub-periods, such as (i) 1985-1987, (ii) 1995-1999 and (iii) 2003-2006 periods. Stock market has been on the receiving end most of the time period except for (i) late 1980s to mid 1990s and (ii) 2007-2011 periods. Bond market is almost always a net receiver of connectedness except for (i) 1994-1999, (ii) short time in 2004 and (iii) 2007-2008 periods. On the other hand, U.S. dollar index is a net receiver for almost all but some relatively shorter periods.

Further details of the direction of the connectedness effects can be elaborated in Figure 3 which presents pairwise directional connectedness measures along with the total (to others/from others) directional and system-wide connectedness measures. It allows us to clearly identify the sources of the connectedness effects. Shocks originating from the real side of the economy has the largest impact on the stock market, followed by the foreign exchange and the bond markets. It's impact on the bond market during the global financial crisis was more pronounced than its impact on the foreign exchange market (see first column in Figure 3).

In the second column of Figure 3, we observe that shocks originating from the stock market directed towards the real sector peak, especially, over the volatile periods in the stock markets (1987 stock market crash, emerging market crises of late 1990s) and in mid2000s, before the outbreak of the global financial crisis. Stock market has relatively stable connectedness impact on the DXY during the 1990s with certain level shifts observed in early 2000s and, DXY has some connectedmess impact on the stock market in a similar pattern as well (see second row, fourth column in Figure 3). The connectedness of the stock market's to the bond market is observed to have an increasing trend from 1995 onwards.

\footnotetext{
${ }^{4}$ In the summer of 2013, Federal Reserve revealed its intentions to gradually end the quantitative easing (QE) program. Financial markets overreacted to this announcement (so-called "Taper Tantrum").
} 

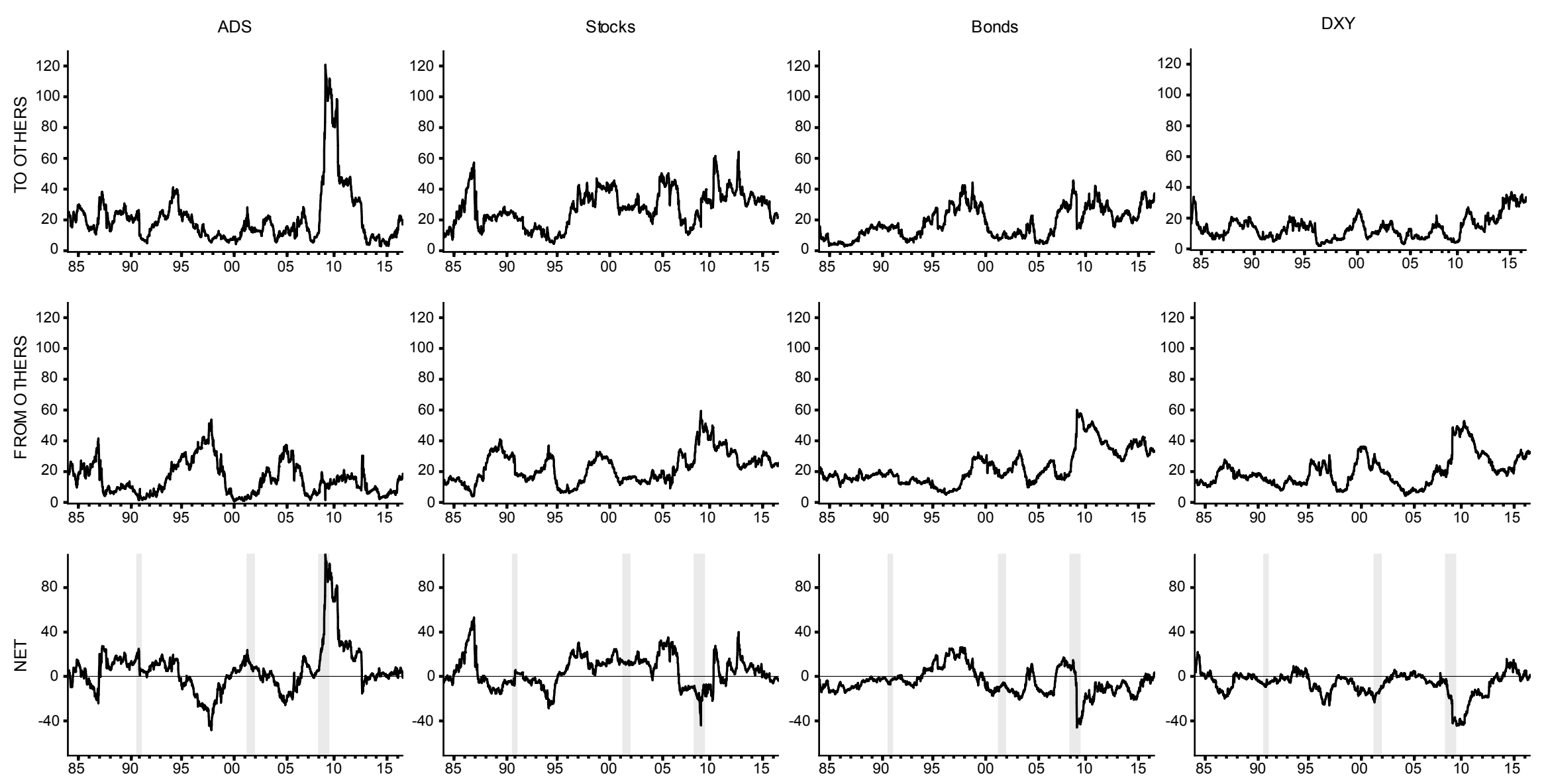

Figure 2: Dynamic total directional connectedness index using ADS 
Shocks from the bonds market to the real side of the economy (see first row, third column in Figure 3) is rather small except some large amount of connectedness observed in the 1994-2000 period. Bond market also gives higher levels of connectedness after 2010 which could be attributed to sovereign debt problems in the EU. In the connectedness from bonds to stock and exchange rate markets, we observe level shifts after 2007.

Among the three financial markets, foreign exchange market turns out to generate the smallest volatility connectedness to other financial markets and the real economy. Connectedness from exchange rate market to the real side of the economy is relatively small except 1991-1996 and 2001-2005 periods.

To sum up, the results presented in Figures 1-3 show that the real side of the U.S. economy (as proxied by the ADS index) generates connectedness towards the financial side (represented by the stock, bond and exchange rate markets). However, before reaching at such a categorical result, we need to take into account that this could be due to the characteristics of the variables used in the analysis. In particular, we should keep in mind that the ADS index is extracted from a factor model of daily yield curve term premium (a financial variable) along with weekly, monthly and quarterly real economic variables. That is to say, the ADS index already incorporates the impact of shocks to financial markets through the yield term premium. As a result, the ADS index is not necessarily a pure measure of real side of the U.S. economy. In order to circumvent this issue, we construct an alternative real activity indicator solely from the real economic variables that are used to extract the ADS index. We repeat our real-financial connectedness analysis and use RAI instead of the ADS index.

\section{An Alternative Weekly Real Activity Index}

To construct a real activity index (RAI) series for the U.S. economy in weekly frequency, we extend a standard monthly frequency factor model. Our model allows for the analysis of missing and mixed-frequency data situations in a common framework. In the literature, similar monthly models have also been promoted by, e.g., Banbura et al. (2011), Banbura and Modugno (2014) and Scotti (2016). 

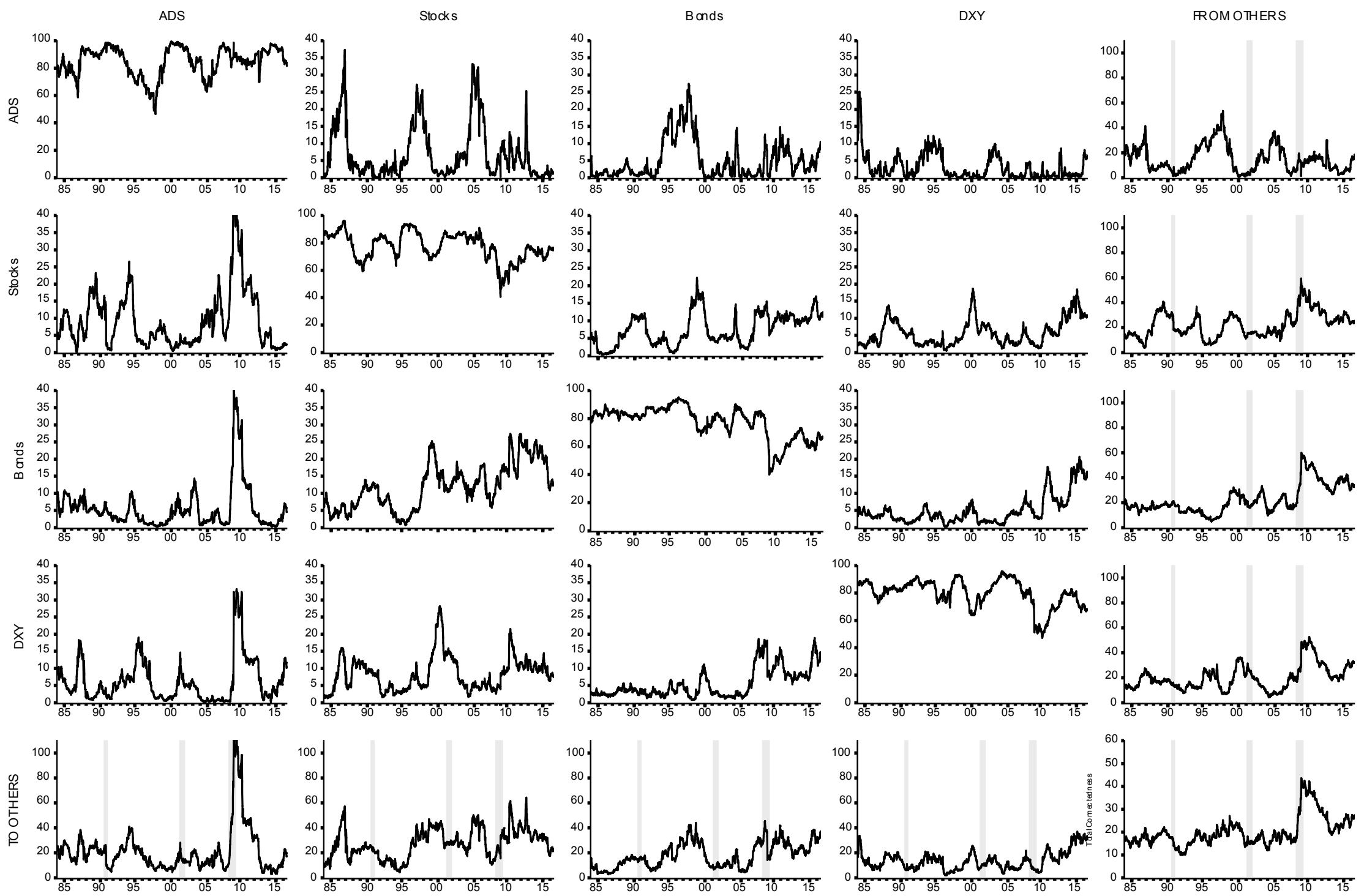
To the best of our knowledge, our work is the first one which extends the abovementioned type of monthly model to a weekly one. Throughout, we mostly follow the modeling conventions used by Scotti (2016). Accordingly; the unobserved factor follows a process of the form:

$$
x_{t+1}=F_{1} x_{t}+\eta_{t}, \eta_{t} \sim \text { i.i.d.N }\left(0, \sigma_{\eta}^{2}\right)
$$

Our model includes 3 variables, weekly $\left(y_{t}^{W}\right)$, monthly $\left(y_{t}^{M}\right)$ and quarterly $\left(y_{t}^{Q}\right)$, which have been transformed to be stationary through suitable transformations and then normalized to have zero mean and unit variance (see Section 5.5 for details). Furthermore; variables are modeled separately.

\subsection{Weekly Variable}

Dynamics of the weekly variable obey the following factor model representation:

$$
y_{t}^{W}=Z^{W} x_{t}+\epsilon_{t}^{W}
$$

The idiosyncratic shocks $\left(\epsilon_{t}^{W}\right)$ follow an $\mathrm{AR}(1)$ process of the form:

$$
\epsilon_{t}^{W}=\alpha_{w} \epsilon_{t-1}^{W}+e_{t}^{W}, e_{t}^{W} \sim \text { i.i.d.N }\left(0, \sigma_{e^{W}}^{2}\right)
$$

In the following, we incorporate quarterly and monthly variables into the model in the spirit of Mariano and Murasawa (2003).

\subsection{Quarterly Variable}

Let $\log$ of quarterly variable, $Y_{t}^{Q}$, be observed at the end of each quarter and be equal to the sum of $\log$ of unobservable weekly components, $Y_{t}^{W}$, and assume that each quarter is comprised of 13 weeks. Under these assumptions, we obtain the following:

$$
Y_{t}^{Q}=Y_{t}^{W}+Y_{t-1}^{W}+Y_{t-2}^{W}+\cdots+Y_{t-12}^{W}
$$

To obtain growth rate for the quarterly variable in (11), we define a partially observed 
weekly series, $y_{t}^{Q}$ :

$$
y_{t}^{Q}= \begin{cases}Y_{t}^{Q}-Y_{t-13}^{Q} & , \text { for } t=13,26,39, \ldots \\ N / A & , \text { otherwise }\end{cases}
$$

We compute $Y_{t}^{Q}-Y_{t-13}^{Q}$ for the variable defined in (12) in terms of (11) and obtain:

$$
Y_{t}^{Q}-Y_{t-13}^{Q}=\underbrace{Y_{t}^{W}-Y_{t-13}^{W}}_{I}+\underbrace{Y_{t-1}^{W}-Y_{t-14}^{W}}_{I I}+\underbrace{Y_{t-2}^{W}-Y_{t-5}^{W}}_{I I I}+\cdots+\underbrace{Y_{t-12}^{W}-Y_{t-25}^{W}}_{X I I I}
$$

$I, I I, \ldots, X I I I$ in $(13)$ can, further, be decomposed into

$$
\begin{gathered}
I: Y_{t}^{W}-Y_{t-13}^{W}=\underbrace{Y_{t}^{W}-Y_{t-1}^{W}}_{y_{t}}+\underbrace{Y_{t-1}^{W}-Y_{t-2}^{W}}_{y_{t-1}}+\cdots+\underbrace{Y_{t-12}^{W}-Y_{t-13}^{W}}_{y_{t-12}} \\
I I: Y_{t-1}^{W}-Y_{t-14}^{W}=\underbrace{Y_{t-1}^{W}-Y_{t-2}^{W}}_{y_{t-1}}+\underbrace{Y_{t-2}^{W}-Y_{t-3}^{W}}_{y_{t-2}}+\cdots+\underbrace{Y_{t-13}^{W}-Y_{t-14}^{W}}_{y_{t-13}} \\
\vdots \\
\vdots \\
X I I I: Y_{t-12}^{W}-Y_{t-25}^{W}=\underbrace{Y_{t-12}^{W}-Y_{t-13}^{W}}_{y_{t-12}}+\underbrace{Y_{t-13}^{W}-Y_{t-14}^{W}}_{y_{t-13}}+\cdots+\underbrace{Y_{t-24}^{W}-Y_{t-25}^{W}}_{y_{t-24}}
\end{gathered}
$$

Defining $y_{t}=\triangle Y_{t}^{W}$; when we add 13 subequations in Equation 14, we obtain the observed quarterly growth rates $\left(Y_{t}^{Q}-Y_{t-13}^{Q}\right)$ in terms of the unobservable weekly growth rates $\left(y_{t}\right)$ which links quarterly series to a weekly one in the spirit of Mariano and Murasawa (2003):

$$
\begin{aligned}
Y_{t}^{Q}-Y_{t-13}^{Q}=y_{t} & +2 y_{t-1}+3 y_{t-2}+4 y_{t-3}+5 y_{t-4}+6 y_{t-5}+7 y_{t-6}+8 y_{t-7}+9 y_{t-8} \\
& +10 y_{t-9}+11 y_{t-10}+12 y_{t-11}+13 y_{t-12}+12 y_{t-13}+11 y_{t-14} \\
& +10 y_{t-15}+9 y_{t-16}+8 y_{t-17}+7 y_{t-18}+6 y_{t-19} \\
& +5 y_{t-20}+4 y_{t-21}+3 y_{t-22}+2 y_{t-23}+y_{t-24}
\end{aligned}
$$

Then, quarterly variable can be represented in weekly frequency as follows:

$$
\begin{aligned}
& y_{t}^{Q}=Z^{Q} x_{t}+\epsilon_{t}^{Q} \\
& \epsilon_{t}^{Q}=\alpha_{q} \epsilon_{t-1}^{Q}+e_{t}^{Q}, e_{t}^{Q} \sim \text { i.i.d.N }\left(0, \sigma_{e^{Q}}^{2}\right)
\end{aligned}
$$




\subsection{Monthly Variable}

For the monthly variable, we use a similar approach that we followed for the quarterly variable. However, since we assumed that each quarter has 13 weeks, it is inevitable that certain months are comprised of 5 weeks instead of 4 . To be able to compute growth rates for the monthly variable, we should consider three different cases that arise due to different number of weeks in consecutive months $[(4,4),(4,5)$ and $(5,4)]$. Consider the notation (4,5): 4 indicates that time period (i.e. week) $t$ is in a 4 -week month and the preceding month has 5 weeks. In the following, we derive relevant equation for each case:

\section{i) $(4,4)$ case:}

Throughout the rest of this section, we follow a slightly modified version of the convention we used for the quarterly variable above. We represent the log of a 4 -week month with $Y_{t}^{M, 4}$ in terms of $\log$ of weekly unobserved components, $Y_{t}^{W}$, as follows:

$$
Y_{t}^{M, 4}=Y_{t}^{W}+Y_{t-1}^{W}+Y_{t-2}^{W}+Y_{t-3}^{W}
$$

With this definition, monthly growth rate $\left(Y_{t}^{M, 4}-Y_{t-4}^{M, 4}\right)$ in weekly frequency can be written as follows:

$$
Y_{t}^{M, 4}-Y_{t-4}^{M, 4}=\underbrace{Y_{t}^{W}-Y_{t-4}^{W}}_{I}+\underbrace{Y_{t-1}^{W}-Y_{t-5}^{W}}_{I I}+\underbrace{Y_{t-2}^{W}-Y_{t-6}^{W}}_{I I I}+\underbrace{Y_{t-3}^{W}-Y_{t-7}^{W}}_{I V}
$$

$I, I I, I I I, I V$ can be written as:

$$
\begin{gathered}
I: Y_{t}^{W}-Y_{t-4}^{W}=\underbrace{Y_{t}^{W}-Y_{t-1}^{W}}_{y_{t}}+\underbrace{Y_{t-1}^{W}-Y_{t-2}^{W}}_{y_{t-1}}+\underbrace{Y_{t-2}^{W}-Y_{t-3}^{W}}_{y_{t-2}}+\underbrace{Y_{t-3}^{W}-Y_{t-4}^{W}}_{y_{t-3}} \\
I I: Y_{t-1}^{W}-Y_{t-5}^{W}=\underbrace{Y_{t-1}^{W}-Y_{t-2}^{W}}_{y_{t-1}}+\underbrace{Y_{t-2}^{W}-Y_{t-3}^{W}}_{y_{t-2}}+\underbrace{Y_{t-3}^{W}-Y_{t-4}^{W}}_{y_{t-3}}+\underbrace{Y_{t-4}^{W}-Y_{t-5}^{W}}_{y_{t-4}} \\
I I I: Y_{t-2}^{W}-Y_{t-6}^{W}=\underbrace{Y_{t-2}^{W}-Y_{t-3}^{W}}_{y_{t-2}}+\underbrace{Y_{t-3}^{W}-Y_{t-4}^{W}}_{y_{t-3}}+\underbrace{Y_{t-4}^{W}-Y_{t-5}^{W}}_{y_{t-4}}+\underbrace{Y_{t-5}^{W}-Y_{t-6}^{W}}_{y_{t-5}} \\
I V: Y_{t-3}^{W}-Y_{t-7}^{W}=\underbrace{Y_{t-3}^{W}-Y_{t-4}^{W}}_{y_{t-3}}+\underbrace{Y_{t-4}^{W}-Y_{t-5}^{W}}_{y_{t-4}}+\underbrace{Y_{t-5}^{W}-Y_{t-6}^{W}}_{y_{t-5}}+\underbrace{Y_{t-6}^{W}-Y_{t-7}^{W}}_{y_{t-6}}
\end{gathered}
$$


When we add subequations in Equation 19, we obtain the following equation which associates unobservable weekly growth rates with monthly growth rates of the observable variables:

$$
Y_{t}^{M, 4}-Y_{t-4}^{M, 4}=y_{t}+2 y_{t-1}+3 y_{t-2}+4 y_{t-3}+3 y_{t-4}+2 y_{t-5}+y_{t-6}
$$

\section{ii) $(4,5)$ case:}

Similar to the previous case, weekly components of monthly variables for 4- and 5-week months can be written as:

$$
\begin{aligned}
& Y_{t}^{M, 4}=Y_{t}^{W}+Y_{t-1}^{W}+Y_{t-2}^{W}+Y_{t-3}^{W}+\mathbf{0} \\
& Y_{t}^{M, 5}=Y_{t}^{W}+Y_{t-1}^{W}+Y_{t-2}^{W}+Y_{t-3}^{W}+Y_{t-4}^{W}
\end{aligned}
$$

With this definition, monthly growth rate $\left(Y_{t}^{M, 4}-Y_{t-4}^{M, 5}\right)$ in weekly frequency can be obtained:

$$
Y_{t}^{M, 4}-Y_{t-4}^{M, 5}=\underbrace{Y_{t}^{W}-Y_{t-4}^{W}}_{I}+\underbrace{Y_{t-1}^{W}-Y_{t-5}^{W}}_{I I}+\underbrace{Y_{t-2}^{W}-Y_{t-6}^{W}}_{I I I}+\underbrace{Y_{t-3}^{W}-Y_{t-7}^{W}}_{I V}+\underbrace{\mathbf{0}-Y_{t-8}^{W}}_{V}
$$

$I, I I, I I I, I V$ are written as in Equation 19, but to be able to apply data to the model, we assume that:

$$
V: \mathbf{0}-Y_{t-8}^{W}=0
$$

Adding up the subequations $I, I I, I I I, I V$ and $V$, we obtain the equation which associates unobservable weekly growth rates with monthly growth rates of observable variables:

$$
Y_{t}^{M, 4}-Y_{t-4}^{M, 5}=y_{t}+2 y_{t-1}+3 y_{t-2}+4 y_{t-3}+3 y_{t-4}+2 y_{t-5}+y_{t-6}
$$

Note that Equation 23 and Equation 20 are equal.

\section{iii) $(5,4)$ case:}

Applying the same approach we used for $(4,4)$ and $(4,5)$ cases, we obtain the following 
equation:

$$
Y_{t}^{M, 5}-Y_{t-5}^{M, 4}=y_{t}+2 y_{t-1}+3 y_{t-2}+4 y_{t-3}+4 y_{t-4}+3 y_{t-5}+2 y_{t-6}+y_{t-7}
$$

Note the slight difference of Equation 24 from Equations 20 and 23, respectively.

For the monthly variable, we construct a partially observed weekly series in the spirit of Equation (12) and obtain the model equations for the monthly variable:

$$
\begin{aligned}
& y_{t}^{M}=Z^{M} x_{t}+\epsilon_{t}^{M} \\
& \epsilon_{t}^{M}=\alpha_{m} \epsilon_{t-1}^{M}+e_{t}^{M}, e_{t}^{M} \sim \text { i.i.d.N }\left(0, \sigma_{e^{M}}^{2}\right)
\end{aligned}
$$

\subsection{Weekly Factor Model}

Stacking weekly, monthly and quarterly variables in $y_{t}=\left(y_{t}^{W}, y_{t}^{M}, y_{t}^{Q}\right)^{\prime}$ vector and combining the equations we have developed so far, we can cast the model into a factor model of the form:

$$
\begin{aligned}
y_{t} & =Z_{t} \alpha_{t} \\
\alpha_{t} & =T \alpha_{t-1}+u_{t}
\end{aligned}
$$

In the weekly model $(26), Z_{t}$ matrix is time-varying due to the varying number of weeks in a month throughout time as we have discussed above. If week $t$ is in a 5-week month and the previous month is a 4 -week month, we use $Z_{t}=Z_{5,4}$. Similarly, if week $t$ is in a 4-week month and the previous month is a 4 - or a 5 -week month, then we use $Z_{t}=Z_{4,4}$. With this setup, we estimate the model and extract the weekly factor from the data which we use as the weekly real activity indicator for the U.S. economy. $Z_{5,4}, Z_{4,4}, \alpha_{t}, T, u_{t}$ and $\Sigma_{u}$, the variance matrix of the shocks hitting the the state vector $\alpha_{t}$, are given in Appendix A.

As documented in more detail in Section 5.5, our model uses three variables, and consequently, the number of unknowns is rather limited. Hence, we are free from most of the complications of the Maximum Likelihood Estimation (MLE) approach that could arise from the use of large number of variables. 
We estimate model (26) using MLE and as an identification restriction, we set factor loading of the GDP variable $\left(Z^{Q}\right)$ to 1 so as to attain close association of the unobserved factor with GDP.

Upon estimation of the model (26) for the U.S., we use Kalman Smoother to extract unobserved factor which is used as a proxy for the real activity of the U.S. economy.

\subsection{Data for RAI}

The variables used to construct RAI are the real variables as in Aruoba et al. (2009). They are; initial claims for unemployment insurance, non-agricultural payrolls and real GDP series which are in weekly, monthly and quarterly frequencies, respectively. Before proceeding to RAI estimation, we suitably transform the data and normalize each series to have zero mean and unit variance. Table 1 provides information about the data used to estimate the weekly factor model underlying RAI.

\begin{tabular}{llcl}
\hline \hline Series & Description & Freq. & Source \\
\hline GDP & Real GDP growth & $\mathrm{Q}$ & FRED \\
Employment & $\Delta$ Nonagricultural employment & $\mathrm{M}$ & FRED \\
Initial Claims & $\Delta$ Initial claims for unemp. insurance & $\mathrm{W}$ & FRED \\
\hline \hline
\end{tabular}

Table 1: Real economic indicators for the U.S.

\section{RAI vs ADS Index}

As we explained in the introduction and throughout the text, our research question pertains to the interaction of the real and financial sides of the U.S. economy at the highest possible frequency. To be able to trace the consequences of the shocks hitting either side of the economy, we need to strictly isolate the real and financial sides of the economy. Since the ADS index uses financial and real variables simultaneously, the resulting index does not exclusively represent the real side of the economy. It is, in a way, "polluted" with information from the financial side of the economy. To isolate the real side of the economy from its financial side, we estimate a "pure" real activity index, RAI, which is solely constructed from the real variables. As no real variable is available at a 
frequency higher than weekly, our real activity indicator is constrained to be in weekly frequency.

Adopting three macroeconomic series employed in the estimation of the ADS index and the model as detailed in Section 5, we estimate a pure real activity indicator for the U.S. We compute RAI for the April 1967 - June 2016 period. We plot both economic activity indicators, RAI and ADS, in Figure $4 .^{5}$ At a first glance, the two series seem to behave quite similarly over time. Yet, when one has closer look at the two series differences between the two become clear. RAI captures peaks and troughs throughout the time period analyzed. It is also important to note that the ADS index is usually leading RAI albeit with a small lead, which could be attributed to the fact that financial data used in extracting the ADS index is usually forward-looking.

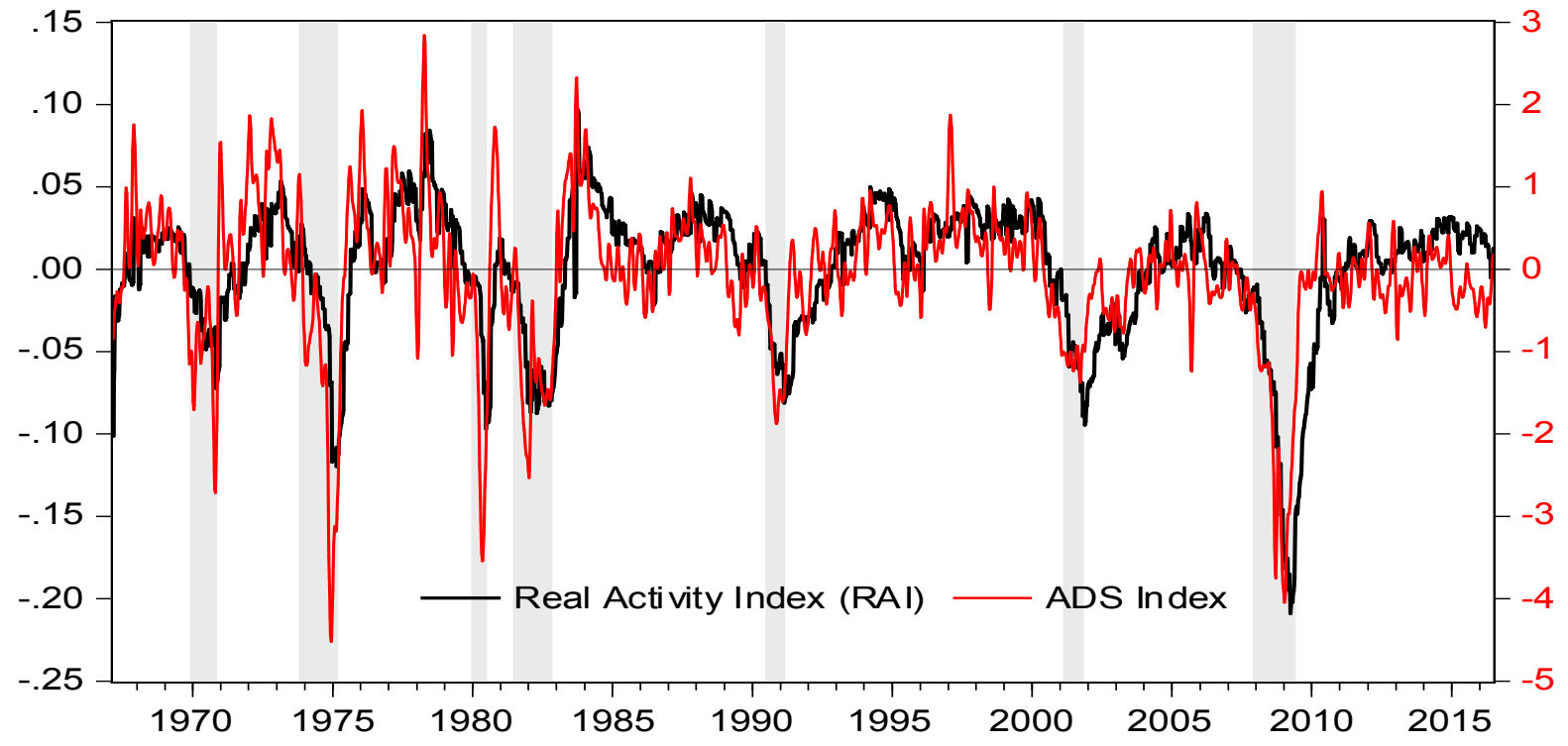

Figure 4: Weekly ADS (right scale) vs RAI (left scale)

Furthermore, in the short-run the ADS index is more volatile than RAI, which follows a relatively smoother path. This can be associated to the use of information on yield curve term premium (the difference between ten-year and three-month Treasury yields) in the calculation of the ADS index. Yield curve term premium fluctuates more than the real variables and hence this translates to more fluctuation in the ADS index than RAI.

\footnotetext{
${ }^{5}$ Shaded regions in the figure represent U.S. recession dates. For the U.S. recession dates, see NBER U.S. Business Cycle Expansions and Contractions, https://nber.org/cycles.html, retrieved 2018-01-28.
} 
In the following, we refrain to discuss the behavior of the two series during important junctures in American economy in detail, not only because that would take too much space but also it is out of the scope of this study. Instead, we think it would be sufficient to have a cursory look at the more recent history of the two series. While RAI fluctuates above level zero most of the time since 2011, the ADS index frequently falls below zero, indicating a contractionary business environment. In particular, the ADS index is consistently below zero since the end of 2013, whereas RAI is always above zero, in line with the observed performance of the U.S. economy.

\section{Measuring Connectedness with RAI}

As in the case of connectedness analysis conducted with the ADS index in Section 4, our connectedness analysis with RAI is based on the estimation of a $\operatorname{VAR}(3)$ model, over 4-year (208-week) rolling sample windows from January 1980 to June 2016. Similarly, we choose a 52-week forecast horizon. In Figure 5(b), we plot the DYCI results with stationary VAR model estimates. To be able to compare the ADS index based and RAI based total connectedness indices, in Figure 5(a), we plot the ADS-based connectedness index again.

A closer look at the two connectedness indices reveals that the RAI-based connectedness index captures financial market shocks better than the ADS-based one. We observe traces of significant economic and political events over our analysis period. And those type of events abound, in particular, during the 1990s; mostly due to volatile economic conditions in the emerging/developed economies and their financial markets. These type of incidences had important connectedness consequences in the form of contagion. As documented in Summers (2000): "Another feature of the 1990s episodes of turmoil is the presence of international 'contagion,' seen in the ERM crisis of 1992-1993, the 'tequila' effects of the Mexican peso crisis of 1994-1995, the 'yellow fever' effects of the Asian crisis of 1997-1998, and the asset-market contagion following the Russian devaluation and default in August 1998 and the Brazilian devaluation in January 1999."

Post October 1987 Black Monday market crash; connectedness index subsides as the impact of October 19, 1987 observation is diluted over the sample window. And on 


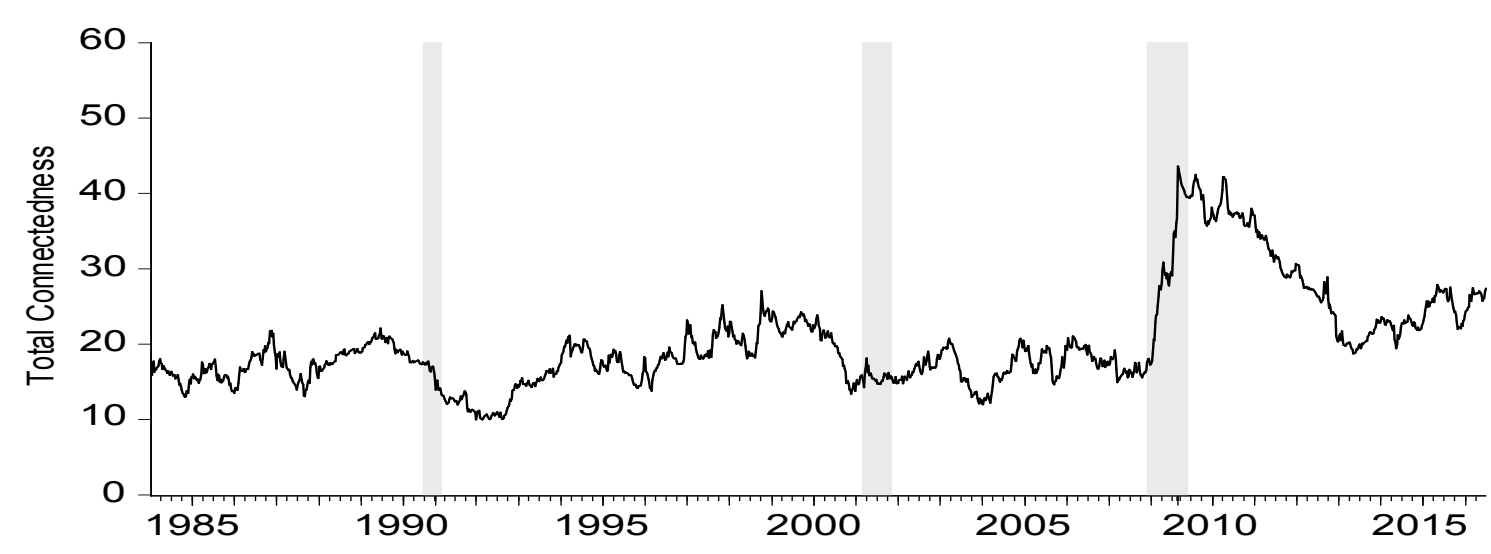

(a) ADS

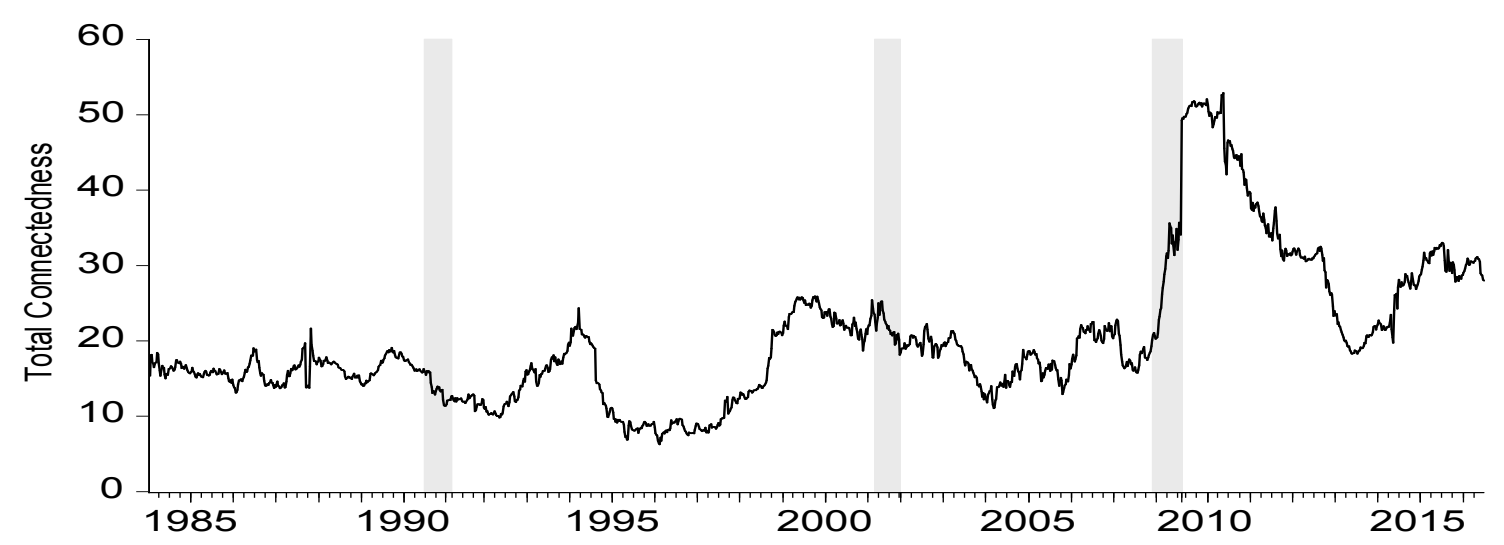

(b) RAI

Figure 5: Total Connectedness Index: ADS vs RAI

October 13, 1989 U.S. stock markets plunged, so called-Friday the 13th mini crash, which marked a local peak in the RAI-based connectedness index. On the real economic side, "1990 marked the end of the longest peacetime expansion in the history of the U.S. economy...", see Walsh (1993). Walsh (1993) identifies Iraq's invasion of Kuwait in 1990 which drove the oil prices up, decreased consumer confidence and FED's attempts to lower inflation rate as the causes of the 1990-1991 recession in the U.S., amongst others. During this period, RAI-based connectedness index declines and rebounds from a local minimum in 1992 to reach a local maximum in 1994 again.

1992-1996 period can be divided into two distinct subperiods: 1992-1994 and 19941996 periods. In the 1992-1994 period; U.S. presidential election took place and Bill Clinton became the new president. In 1992-1993, financial turmoil hit the international foreign exchange markets; UK had to withdraw GBP from the Exchange Rate Mechanism 
(ERM) as it was not able to meet ERM requirements set forth. In 1993, the crisis has resurfaced again effecting several other currencies and the issue is resolved in August 1993 by an extensive restructuring in the requirements. Domestically, the U.S. economy started to recover from the recession and entered a new growth phase.

In January 1994, NAFTA Agreement came into force between Canada, Mexico and the U.S. Throughout the year, Mexico had political and economic troubles, and then required funding from the U.S. U.S. policymakers feared that "[Mexico] is a country, being on our border, in which serious financial instability would have a very definite possibility of spreading across the border and creating problems in our own markets...", see Sablik (2017). This fear is also reflected in the connectedness index. It peaks in early 1994 and then starts to decrease until mid-1995 to 1996.

Connectedness index starts to increase during the Asian (1997-1998), the Russian (1998) crises. Following the collapse of LTCM (1998), connectedness index more than doubles and then follows a mild downward slope with a jump because of the burst of the dot.com bubble started at the end of March 2000 and affected the major U.S. stock indices towards the end of the year, and led to a recesion starting in March 2001. This is reflected as a small blip in the RAI-based connectedness index. The ensuing downward trend in the index intensifies over the 2003-2004 period during the 2003 Iraq war and the interest rate increases by Greenspan's FED. We also observe a jump in the connectedness index at the end of 2005 , from a level lower than $15 \%$ to a level close to $25 \%$.

The connectedness index explodes and reaches all-time-high levels with the the Global Financial Crisis and the subsequent European sovereign debt crisis.

It then decreases to pre-crises levels by May 2013. However, as we discussed before, financial markets' response to Fed's decision to progressively taper the bond purchases (Taper Tantrum) in June 2013 gradually moved the index upwards. Reflecting this reaction, the connectedness index increased several percentage points to move close to $30 \%$. U.S. bond market flash crash in October 2014 as well as the the increased worries about the Chinese shadow financial institutions in the summer of 2015 led the index to increase above $30 \%$ level.

Even though the connectedness index as of the end of the analysis period (June 2016) is lower than its peak in 2009-2010, it is nevertheless higher than the levels it attained 
during the tranquil periods of the pre-crisis period. Unlike the previous episodes, the two financial crises (U.S. and European) had permanent impact on the real-financial connectedness.

A similar observation was made by Demirer et al. (2018) in a completely global financial context: Once the global financial crisis hit, connectedness among the globally systemically important banks (GSIBs) moved to a higher level.

Figures 6 and 7 present the dynamic total and pairwise directional connectedness measures, respectively. Unlike the results we obtained with ADS index, when we use RAI in the real and financial connectedness estimations, the real side of the economy appears to be on the receiving end most of the time. 1984-1987 and 2006-2009 periods are the two main exceptions where it acts as a net connectedness source (Figure 6, NET row, RAI column). At other times, net connectedness of RAI stays in the negative territory except for short time periods. But this does not change our main finding that the real side receives connectedness from the financial side over the analysis period. This is completely different from the results obtained when we used the ADS index in the connectedness analysis.

Overall, stock market appears to be a net transmitter of shocks during almost all of the analysis period (Figure 6, NET row, Stocks column). Two main exceptions to this observation are the 1984-1987 and the 2004-2008 periods. Figure 7 hints us that the real side of the economy is mostly the source of connectedness over the two periods (Figure 7, Stocks row, RAI column). Their roles are switched during the rest of the periods.

10-year government bond volatility has negative levels of net connectedness, with three periods during which its net connectedness moving into the low positive territory. These periods are 2000-2001, 2003-2006 and 2007-2009 periods (Figure 6, NET row, Bonds column). The characteristics of the bond market as a source of connectedness to RAI have changed beginning with year 2000 (Figure 7, RAI row, Bonds column). It has become more of a net source of connectedness to RAI compared to pre-2000 period.

Foreign exchange market (as represented by the U.S. dollar index volatility in our framework) behaves both as a generator and receiver of connectedness but at relatively small magnitudes: during the 1984-1987 and 2014-2016 periods, it behaves as a net connectedness source whereas over the 1999-2001 and the 2007-mid 2010 periods it is 

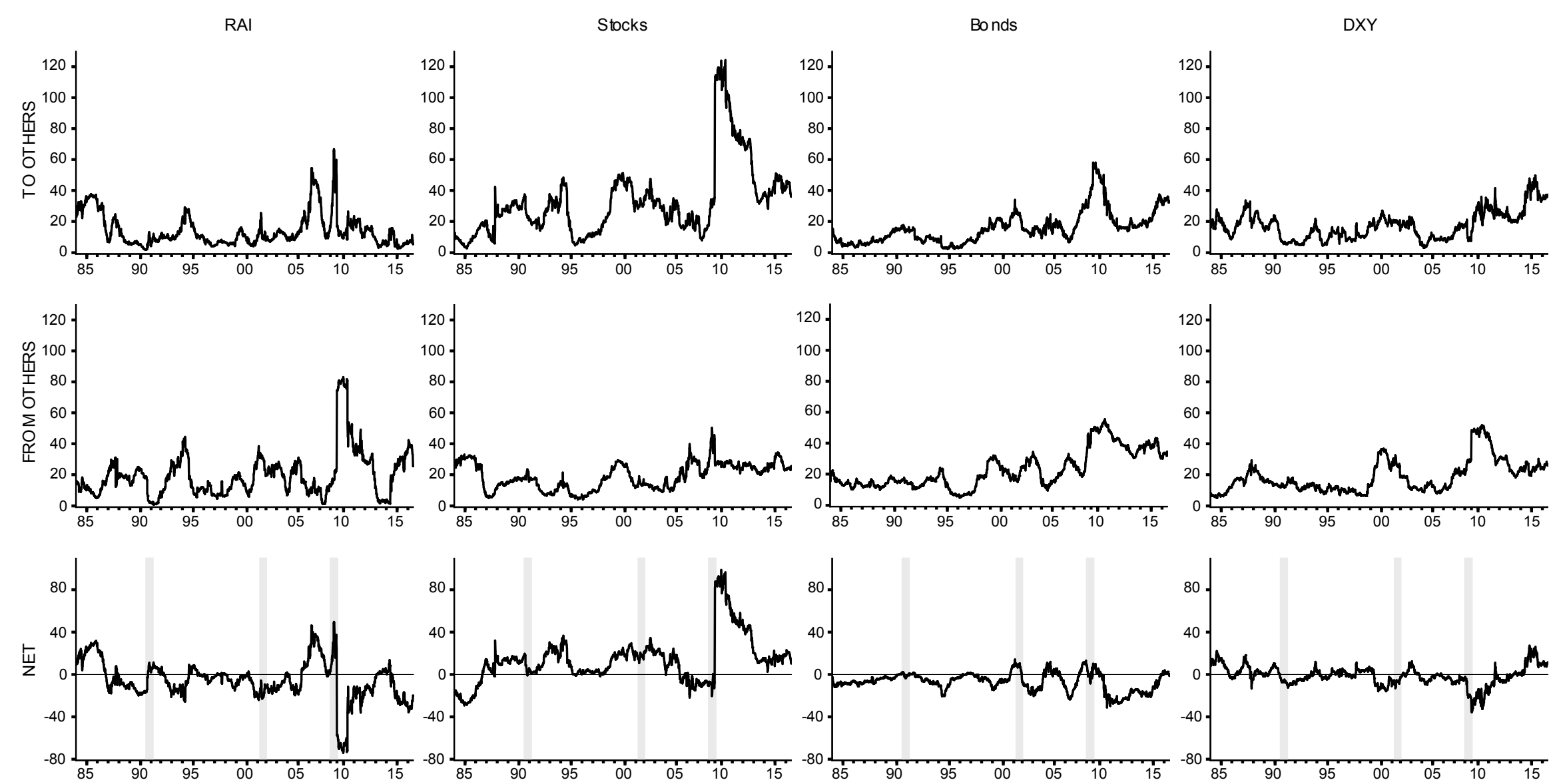

Figure 6: Dynamic Total Directional Connectedness Index using RAI 
much more on the receiving side (Figure 6, NET row, DXY column).

Volatility shocks hitting the financial markets, and especially the stock market, generate net positive connectedness on the real side of the U.S. economy, followed by the bond market.

\section{Connectedness with RAI vs the ADS index}

We have seen in Sections 4 and 7 that the ADS index and RAI, respectively, lead to different conclusions with respect to the interaction of the real and financial sides of the U.S. economy. When we use the ADS index to represent the real side of the economy, we found that the real side of the economy effects financial side significantly. However, when we use RAI to represent the real side, the reverse is observed, i.e. financial side effects the real side prominently. Shocks to financial side have important consequences on the real side. In this section, we compare connectedness indices for both analyses which is presented in Figure 5.

Even though the details and the implications of both analyses differ, on average, both connectedness indices display similar patterns. In particular, we can identify three bulges common to both analyses, corresponding to the periods: (i) late 1980s to early 1990s (due to Black Monday (1987), Friday the 13th mini crash (1989), U.S. recession in 1990-1991), (ii) mid 1990s to early 2000s (due to Mexican (1994), Asian (1997-1998) and Russian (1998) crises, the collapse of LTCM (1998), U.S. recession in 2001 and the September 11 terrorist attacks (2001)), and (iii) late 2000s to early 2010s (due to global financial (2007-2009) and the EU sovereign debt crises (beginning in 2010)). However, RAI-based analysis provides finer details over the analysis period. Another important observation is that connectedness index for the analysis with RAI, see Figure 5(b), is more volatile than that of the ADS index, see Figure 5(a). This observation could possibly be attributed to the inclusion of a financial variable in the estimation of the ADS index. RAI, which is "purely" estimated from real variables, could be more prone to financial shocks than the ADS index. 

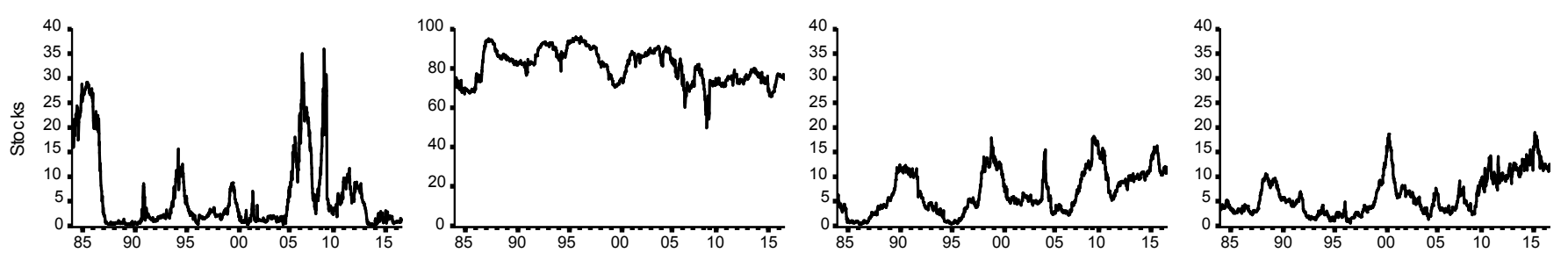

$\frac{{ }_{85}}{20.4}$
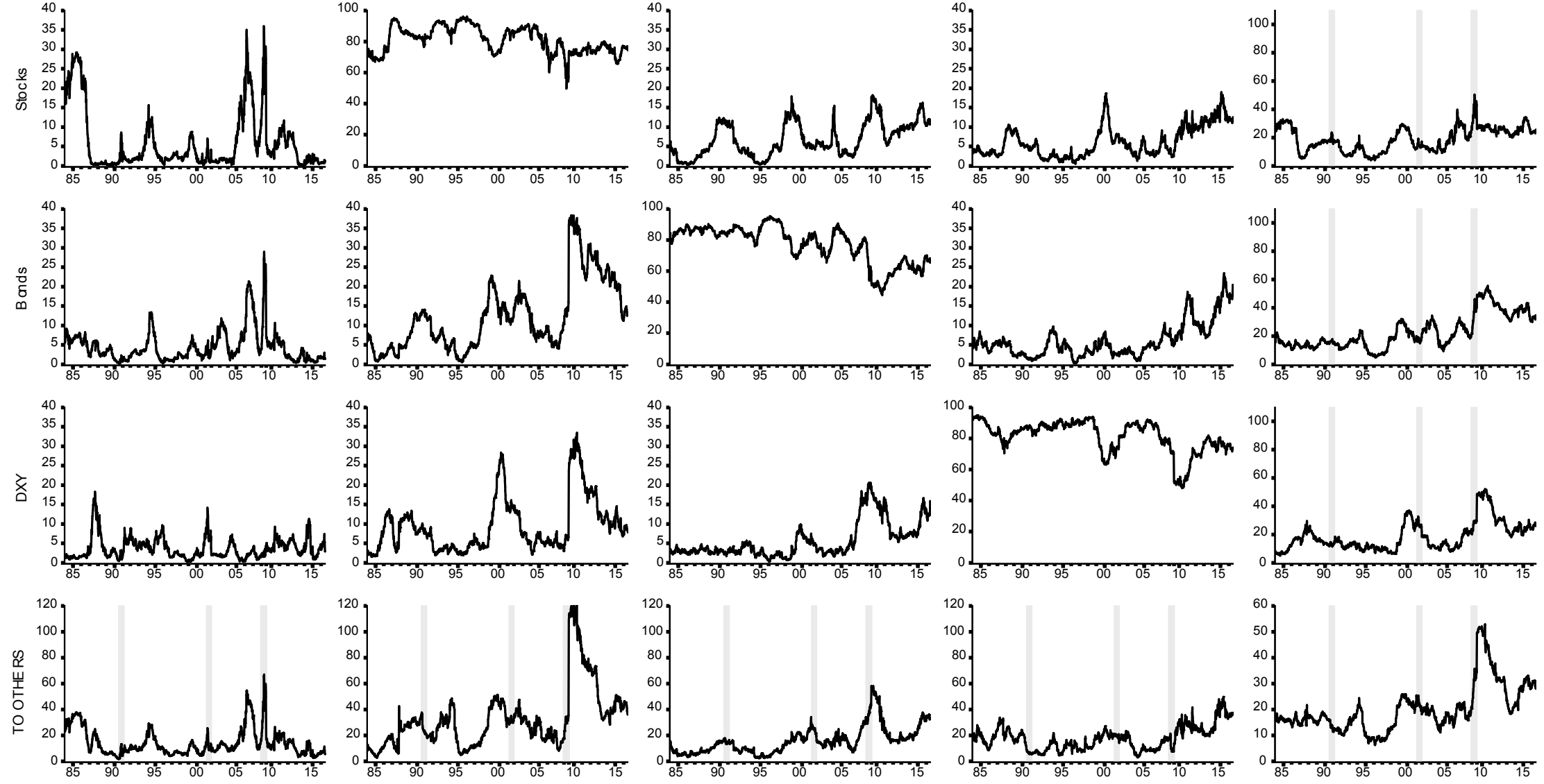
To further elaborate the similarities and the dissimilarities of the ADS index based and RAI-based analyses, we plot the net directional connectedness from the real side of the economy to respective financial markets, for the ADS index in Figure 8(a) and for RAI in Figure 8(b) (note that in Figure 6, we plot net connectedness generated from all other nodes of our network to respective nodes). In the leftmost panel of Figure 8(a), we observe that connectedness from ADS to stock market is positive most of the time except for some short time periods. Same holds for the bond market as well, see the mid panel in Figure 8(a). However, in the case of the foreign exchange rate we observe both large positive and small negative values of net connectedness for extended periods, leading to no clear-cut result, see the rightmost panel in Figure 8(a).

Similarly, when we analyse net connectedness effects from RAI to financial markets, we observe that RAI is a net receiver from the stock market except for 1984-1987, 19951999 and 2006-2008 periods, see the leftmost panel in Figure 8(b). For the bond market, there are periods for which RAI acts both as a net source and a net receiver, see the mid panel in Figure 8(b). But especially during the 2008-2012 period, it is significantly a net receiver. For the foreign exchange market, it can be said that RAI is a net receiver for extended periods. At times when it acts as a net source, its impact is rather small, see the rightmost panel in Figure 8(b).

\section{Conclusion}

In this study, we have investigated how shocks to the real and financial sides of the U.S. economy spill over to each other. To that end; we first conducted a DYCI analysis using the ADS index of Aruoba et al. (2009) to represent the real side of the U.S. economy. We represented the financial side by the weekly range-based volatilities for the stock, the bond and the foreign exchange market returns. The ADS index coupled with market return volatilities are fed into the Diebold-Yilmaz framework and allowed us to track shock dynamics and the interactions of the real and financial sectors of the U.S. economy. Through this analysis, we find that shocks to the real side of the economy have important connectedness consequences on the financial side. 

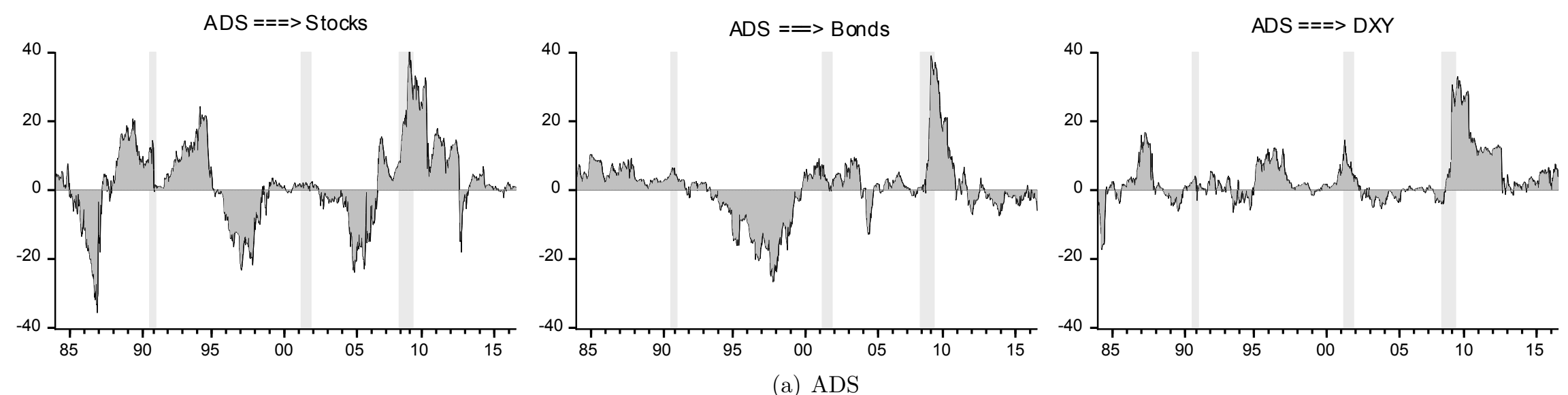

(a) $\mathrm{ADS}$
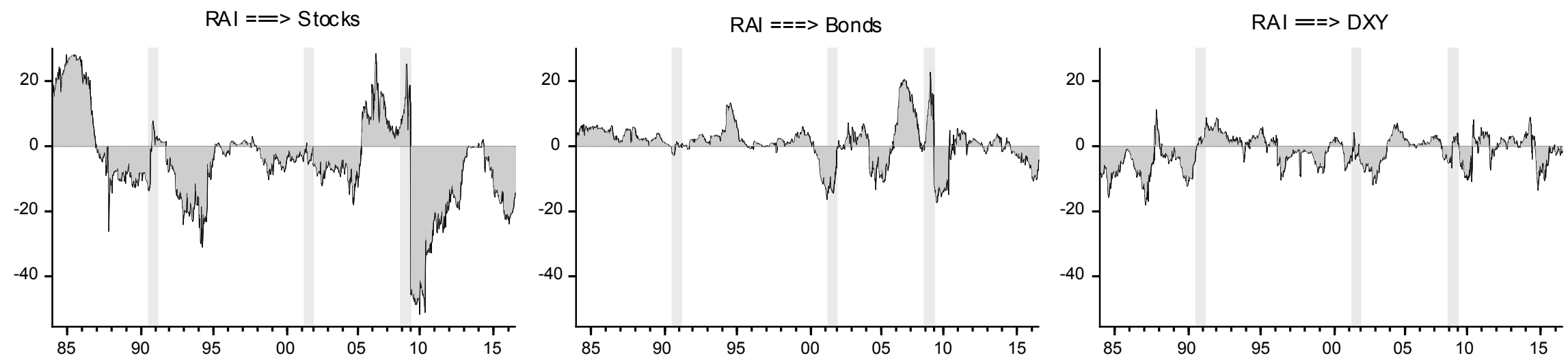

(b) RAI

Figure 8: Net Connectedness from Real to Financial Sector: ADS vs RAI 
One criticism to the use of the ADS index to represent the real side of the economy would be the use of a financial variable in the estimation of the ADS index. To overcome this sort of criticism, we estimate a dynamic factor model of the real side of the economy to obtain a weekly real activity index (RAI) in the spirit of Aruoba et al. (2009).

With the real activity index approach we employ, we create a series which distills information from the same set of macroeconomic variables used in Aruoba et al. (2009) that have different observational frequencies. This mismatch in observational frequencies inevitably leads to missing data but our factor model handles this issue flawlessly and makes use of all available information in the data.

When we compare RAI and the ADS index of Aruoba et al. (2009), we find that the two indicators behave quite similarly over time, the ADS index being more volatile than RAI, a result that could be due to the use of financial data in the ADS index computations.

It could be argued that industrial production index (IPI) is an instrumental proxy for the real activity of an economy in monthly frequency and can be used as an input for the DYCI analysis. This argument could be deemed true up to a certain extent but IPI is not a direct counterpart of the economic activity in the economy. A single index generated from a blended set of variables, such as RAI, reflects the real side of the economy more precisely. Another issue arising from the use of monthly observations in the VAR model would be that depending on the number of variables in the model, reliable estimation results require a 8-10 years of rolling sample window size. Over such a long time frame, the economy could have been hit by many fundamentally different shocks, generating inconclusive results. To make sure that our empirical analysis produces meaningful and interpretable results, we conducted our connectedness analysis in a weekly frequency. To the best of our knowledge, we are the first who extend the commonly used type of monthly factor model to a weekly one and estimate a pure weekly real activity index.

Our analysis with RAI, when used to represent the real side of the economy, suggests that the real and financial sides of the U.S. economy interact significantly, especially, during the emerging market crises of the late-1990s and the global financial crisis. Stock market volatilities have the most impact on the real activity of the U.S. economy followed by the bonds and the foreign exchange markets during these periods. Except for certain 
periods, e.g. 1984-1987,1995-1999 and 2006-2008, real economy is a net receiver of shocks originating from the financial markets.

In line with the literature, our results suggest that the real and financial sides of the economy interact continuously and shocks on one side of the economy have direct and important consequences on the other. 


\section{References}

Acemoglu, D., V.M. Carvalho, A. Ozdaglar, and A. Tahbaz-Salehi (2012), "The Network Origins of Aggregate Fluctuations," Econometrica, 80, 1977-2016.

Acemoglu, D., A. Ozdaglar, and A. Tahbaz-Salehi (2015), "Systemic Risk and Stability in Financial Networks," American Economic Review, 105, 564-608.

Acharya, Viral V., Lasse H. Pedersen, Thomas Philippon, and Matthew Richardson (2010), "Measuring systemic risk," Federal Reserve Bank of Cleveland Working Paper 1002.

Adrian, T. and M. Brunnermeier (2011), "CoVaR," NBER Working Paper 17454.

Anand, K., P. Gai, S. Kapadia, S. Brennan, and M. Willison (2013), "A network model of financial system resilience," Journal of Economic Behavior and Organization, 85, $219-235$.

Aruoba, S.B., F.X. Diebold, and C. Scotti (2009), "Real Time Measurement of Business Conditions," Journal of Business and Economic Statistics, 27, 417-427.

Banbura, M., Domenico Giannone, and Lucrezia Reichlin (2011), "Nowcasting," in "Oxford Handbook on Economic Forecasting," (edited by Clements, Mike P. and David F. Hendry), 193-224, Oxford: Oxford University Press.

Banbura, M. and Michele Modugno (2014), "Maximum Likelihood Estimation of Factor Models on Datasets With Arbitrary Pattern of Missing Data," Journal of Applied Econometrics, 29, 133-160.

Barattieri, Alessandro, Maya Eden, and Dalibor Stevanovic (2013), "The connection between Wall Street and Main Street : measurement and implications for monetary policy," The World Bank Working Paper 6667.

Billio, M., M. Getmansky, A.W. Lo, and L. Pelizzon (2012), "Econometric Measures of Connectedness and Systemic Risk in the Finance and Insurance Sectors," Journal of Financial Economics, $104(3), 535-559$. 
Borio, Claudio and Mathias Drehmann (2011), "Toward an Operational Framework for Financial Stability: "Fuzzy" Measurement and Its Consequences," in "Financial Stability, Monetary Policy, and Central Banking," (edited by Alfaro, Rodrigo), Volume 15 of Central Banking, Analysis, and Economic Policies Book Series, chap. 4, 63-123, Central Bank of Chile.

Brunnermeier, M., G. Gorton, and A. Krishnamurthy (2011), "Risk Topography," NBER Macroeconomics Annual 2011, 26(1), 149-176.

Burns, A.F. and W.C. Mitchell (1946), Measuring Business Cycles, National Bureau of Economic Research.

Cotter, J., M. Hallam, and K. Yilmaz (2017), "Mixed-Frequency Macro-Financial Spillovers," Koç University-TUSIAD Economic Research Forum Working Paper, No. 1704, February.

Demirer, M., F.X. Diebold, L. Liu, and K. Yilmaz (2018), "Estimating Global Bank Network Connectedness," Journal of Applied Econometrics.

Diebold, F. X. and K. Yilmaz (2015), "Measuring the Dynamics of Global Business Cycle Connectedness," in "Unobserved Components and Time Series Econometrics: Essays in Honor of Andrew C. Harvey," (edited by Koopman, S.J. and N. Shephard), 45-70, Oxford University Press.

Diebold, F.X. and K. Yilmaz (2009), "Measuring Financial Asset Return and Volatility Spillovers, with Application to Global Equity Markets," Economic Journal, 119, 158171.

Diebold, F.X. and K. Yilmaz (2012), "Better to Give than to Receive: Predictive Measurement of Volatility Spillovers," International Journal of Forecasting, 28, 57-66.

Diebold, F.X. and K. Yilmaz (2014), "On the Network Topology of Variance Decompositions: Measuring the Connectedness of Financial Firms," Journal of Econometrics, $182,119-134$. 
Dungey, M., M. Luciani, and D. Veredas (2013), "Googling SIFIs," Manuscript, ECARES, Free Université libre de Bruxelles.

Engle, R.F. and B.T. Kelly (2012), "Dynamic Equicorrelation," Journal of Business and Economic Statistics, 30, 212-228.

Gai, P., A. Haldane, and S. Kapadia (2011), "Complexity, concentration and contagion," Journal of Monetary Economics, 58, 453-470.

Gai, P. and S. Kapadia (2010), "Contagion in financial networks," Proceedings of the

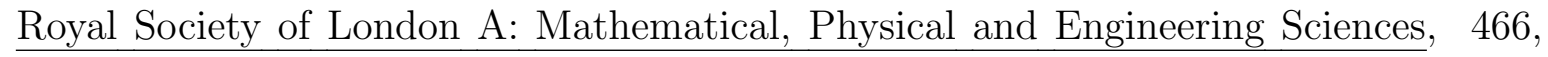
2401-2423.

He, Z. and A. Krishnamurthy (2012), "A model of capital and crises," The Review of Economic Studies, 79, 735-777.

Koop, G., M.H. Pesaran, and S.M. Potter (1996), "Impulse Response Analysis in Nonlinear Multivariate Models," Journal of Econometrics, 74, 119-147.

Mariano, R.S. and Y. Murasawa (2003), "A New Coincident Index of Business Cycles Based on Monthly and Quarterly Series," Journal of Applied Econometrics, 18, 427443.

Markose, S., S. Giansante, and A.R. Shaghaghi (2012), "Too interconnected to fail financial network of US CDS market: Topological fragility and systemic risk," Journal of Economic Behavior \& Organization, 83, 627-646.

Pesaran, H.H. and Y. Shin (1998), "Generalized Impulse Response Analysis in Linear Multivariate Models," Economics Letters, 58, 17-29.

Sablik, Timothy (2017), "The Fed's Tequila Crisis," Federal Reserve Bank of Richmond-Econ Focus, 3-5.

Scotti, C. (2016), "Surprise and Uncertainty Indexes: Real-time Aggregation of Realactivity Macro-surprises," Journal of Monetary Economics, 82, 1-19. 
Stock, J.H. and M.W. Watson (1989), "New Indexes of Coincident and Leading Economic Indicators," NBER Macroeconomics Annual, 4, 351-394.

Stock, J.H. and M.W. Watson (1991), "A Probability Model of The Coincident Economic Indicators," in "Leading Economic Indicators: New Approaches and Forecasting Records," (edited by Lahiri, K. and G. Moore), 63-85, Cambridge University Press.

Summers, Lawrence H. (2000), "International Financial Crises: Causes, Prevention, and Cures," The American Economic Review, 90, 1-16.

Walsh, Carl E. (1993), "What caused the 1990-1991 recession?” Economic Review, 33-48. 


\section{Appendices}

A Model Matrices

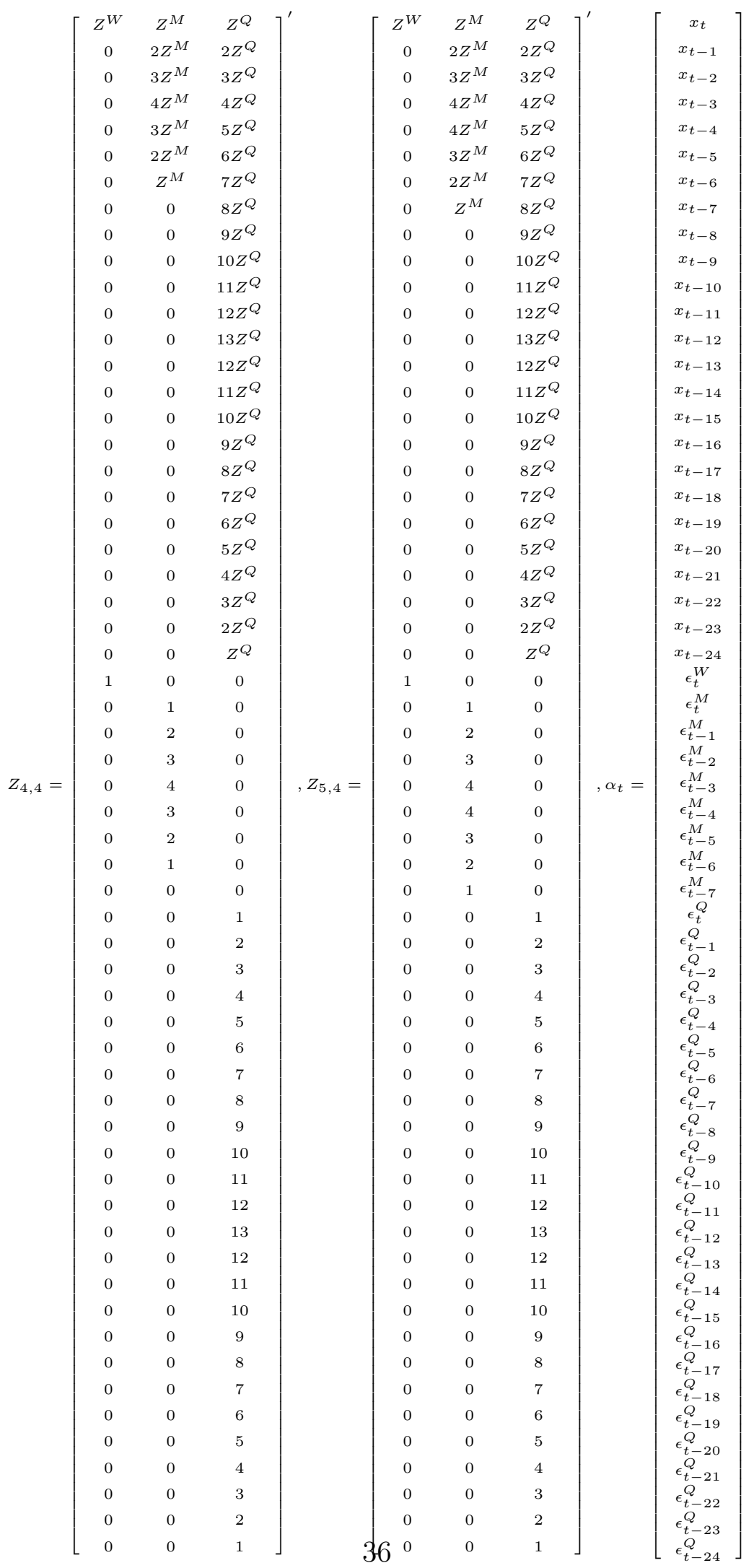




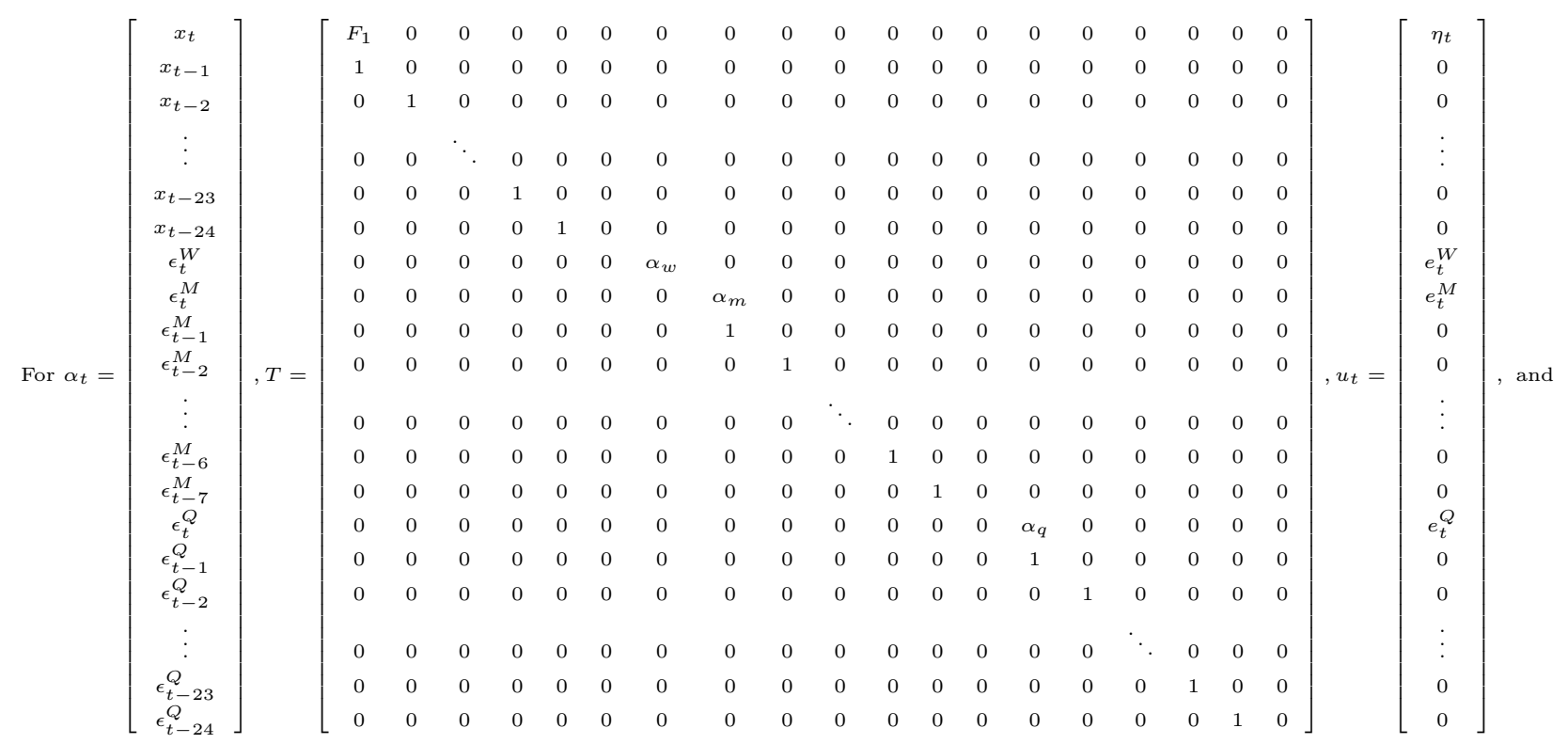

$\Sigma_{u}=\left[\begin{array}{ccccccccccccccccccc}\sigma_{\eta}^{2} & 0 & 0 & 0 & 0 & 0 & 0 & 0 & 0 & 0 & 0 & 0 & 0 & 0 & 0 & 0 & 0 & 0 & 0 \\ 0 & 0 & 0 & 0 & 0 & 0 & 0 & 0 & 0 & 0 & 0 & 0 & 0 & 0 & 0 & 0 & 0 & 0 & 0 \\ 0 & 0 & 0 & 0 & 0 & 0 & 0 & 0 & 0 & 0 & 0 & 0 & 0 & 0 & 0 & 0 & 0 & 0 & 0 \\ 0 & 0 & 0 & 0 & 0 & 0 & 0 & 0 & 0 & 0 & 0 & 0 & 0 & 0 & 0 & 0 & 0 & 0 & 0 \\ 0 & 0 & 0 & 0 & \ddots & 0 & 0 & 0 & 0 & 0 & 0 & 0 & 0 & 0 & 0 & 0 & 0 & 0 & 0 \\ 0 & 0 & 0 & 0 & 0 & 0 & 0 & 0 & 0 & 0 & 0 & 0 & 0 & 0 & 0 & 0 & 0 & 0 & 0 \\ 0 & 0 & 0 & 0 & 0 & 0 & \sigma_{W}^{2} & 0 & 0 & 0 & 0 & 0 & 0 & 0 & 0 & 0 & 0 & 0 & 0 \\ 0 & 0 & 0 & 0 & 0 & 0 & 0 & \sigma_{M}^{2} & 0 & 0 & 0 & 0 & 0 & 0 & 0 & 0 & 0 & 0 & 0 \\ 0 & 0 & 0 & 0 & 0 & 0 & 0 & 0 & 0 & 0 & 0 & 0 & 0 & 0 & 0 & 0 & 0 & 0 & 0 \\ 0 & 0 & 0 & 0 & 0 & 0 & 0 & 0 & 0 & \ddots & 0 & 0 & 0 & 0 & 0 & 0 & 0 & 0 & 0 \\ 0 & 0 & 0 & 0 & 0 & 0 & 0 & 0 & 0 & 0 & 0 & 0 & 0 & 0 & 0 & 0 & 0 & 0 & 0 \\ 0 & 0 & 0 & 0 & 0 & 0 & 0 & 0 & 0 & 0 & 0 & 0 & 0 & 0 & 0 & 0 & 0 & 0 & 0 \\ 0 & 0 & 0 & 0 & 0 & 0 & 0 & 0 & 0 & 0 & 0 & 0 & 0 & 0 & 0 & 0 & 0 & 0 & 0 \\ 0 & 0 & 0 & 0 & 0 & 0 & 0 & 0 & 0 & 0 & 0 & 0 & 0 & \sigma & 0 & 0 & 0 & 0 & 0 \\ 0 & 0 & 0 & 0 & 0 & 0 & 0 & 0 & 0 & 0 & 0 & 0 & 0 & 0 & 0 & 0 & 0 & 0 & 0 \\ 0 & 0 & 0 & 0 & 0 & 0 & 0 & 0 & 0 & 0 & 0 & 0 & 0 & 0 & 0 & \ddots & 0 & 0 & 0 \\ 0 & 0 & 0 & 0 & 0 & 0 & 0 & 0 & 0 & 0 & 0 & 0 & 0 & 0 & 0 & 0 & 0 & 0 & 0 \\ 0 & 0 & 0 & 0 & 0 & 0 & 0 & 0 & 0 & 0 & 0 & 0 & 0 & 0 & 0 & 0 & 0 & 0 & 0 \\ 0 & 0 & 0 & 0 & 0 & 0 & 0 & 0 & 0 & 0 & 0 & 0 & 0 & 0 & 0 & 0 & 0 & 0 & 0\end{array}\right]$ 